\title{
The Prognostic Value of Peripheral Artery Diseases in Patients with ST-Segment Elevation Myocardial Infarction
}

\author{
Olga L. Barbarash, ${ }^{1,2}$ Mikhail V. Zykov, ${ }^{1,2}$ Tamara B. Pecherina, ${ }^{1,2}$ Vasily V. Kashtalap, \\ Leonid S. Barbarash, ${ }^{3}$ and Anton G. Kutikhin ${ }^{4}$ \\ ${ }^{1}$ Research Institute for Complex Issues of Cardiovascular Diseases under the Siberian Branch of the Russian Academy of \\ Medical Sciences, Sosnovy Bulvar 6, Kemerovo 650002, Russia \\ ${ }^{2}$ Department of Cardiology and Cardiovascular Surgery, Kemerovo State Medical Academy, Sosnovy Bulvar 6, \\ Kemerovo 650002, Russia \\ ${ }^{3}$ Kemerovo Cardiological Centre, Sosnovy Bulvar 6, Kemerovo 650002, Russia \\ ${ }^{4}$ Department of Epidemiology, Kemerovo State Medical Academy, Voroshilova Street 22a, Kemerovo 650002, Russia
}

Correspondence should be addressed to Olga L. Barbarash; barbol@cardio.kem.ru

Received 28 August 2013; Revised 10 November 2013; Accepted 18 November 2013

Academic Editor: Francisco Blanco-Vaca

Copyright ( 2013 Olga L. Barbarash et al. This is an open access article distributed under the Creative Commons Attribution License, which permits unrestricted use, distribution, and reproduction in any medium, provided the original work is properly cited.

Hypothesis. To evaluate the clinical and prognostic role of haemodynamically insignificant stenosis of the extracranial arteries (ECA) and lower extremity arteries (LEA) among patients with ST-segment elevation myocardial infarction (STEMI). Patients and Methods. The study sample consisted of 423 patients with STEMI who were consecutively admitted to the Kemerovo Cardiological Centre. Results. The prevalence of polyvascular diseases (PVD), as defined by an increased intima-media thickness (IMT) of the common carotid artery or by stenosis of the ECA or LEA, was 95\%. Among patients with ECA or LEA, the case fatality rate of those with stenosis with occlusion of less than $30 \%$ of the vessel lumen was $5.7 \%$, whereas the case fatality rate among patients with stenosis with occlusion of more than $30 \%$ of the vessel lumen was $15.1 \%\left(\chi^{2}=13.68, P=0.003\right)$. Using the GRACE score model, together with the determination of additional factors (congestive heart failure, PVD, prior stroke, and smoking status), we developed an improved model (KemScore) for death risk stratification for a 12-month period. The value of the AUC for our model (KemScore) was $0.83(95 \% \mathrm{CI}=0.76-0.90)$, which was significantly higher than the initial GRACE score value of $0.71(95 \% \mathrm{CI}=$ $0.63-0.79)$.

\section{Introduction}

Accurate risk stratification plays a very important role in the management of patients with acute and chronic coronary heart diseases (CHD). However, current prediction models that determine the potential adverse outcomes in patients with acute coronary syndromes do not take coronary artery lesions into account [1-3]. It is also well known that atherosclerosis is a systemic pathological process affecting multiple arteries (coronary, extracranial, and arteries of the lower extremities). Much of the current research in this area has focused on local manifestations of atherosclerosis in the affected area, leaving lesions of the associated vascular regions largely unnoticed due to the lack of proper diagnostic evaluation methods. Stenosis of the coronary and cerebral arteries may manifest clinically as acute cardiovascular events or stroke, respectively, whereas other vascular lesions appear to be latent and may result in fatal complications under certain conditions. The choice of treatment method is of critical importance in patients with subclinical atherosclerosis in combination with CHD because many of these conditions are characterised by an absence of haemodynamically significant lesions of noncoronary arteries. Patients with polyvascular diseases (PVD) are at a high risk, requiring a special approach in treatment due to the characteristics of the arterial lesions [4]. This study evaluates the clinical and prognostic role of haemodynamically insignificant stenosis of the extracranial and lower extremity arteries among patients with ST-segment 
elevation myocardial infarction (STEMI). We attempted to develop a method for improving the predictive value of GRACE risk score by adding the additional risk factors.

\section{Materials and Methods}

2.1. Study Design. The study sample consisted of 423 Russian patients with ST-segment elevation myocardial infarction (STEMI) consecutively admitted to the Kemerovo Cardiological Centre in 2009. All the patients were the residents of Kemerovo city. In compliance with the recommendations of the European Society of Cardiology [5], the inclusion criterion for this study was a diagnosis of STEMI up to 24 hours after its onset. Age under 18 years and myocardial infarction after the percutaneous coronary intervention or coronary artery bypass surgery were the exclusion criteria.

2.2. Details of Study Protocol. This study was approved by the local ethical committee, and all patients signed informed consent forms. Complaints, previous medical history, results of clinical examination, electrocardiogram data, levels of cardiospecific enzymes, systemic haemodynamic values, and echocardiogram data were registered. Colour duplex screening of the extracranial arteries (ECA) and lower extremity arteries (LEA) was performed on 5-7 days of hospitalization for all patients using the cardiovascular ultrasound system Vivid 7 Dimension (General Electric, USA) with a $5.7 \mathrm{MHz}$ linear array transducer (for ECA), a 2.5-3 MHz curved array transducer, and a $5 \mathrm{MHz}$ linear array transducer (for LEA). The extent of arterial stenosis was assessed in $B$ regimen and by dopplerography (visualizing the local haemodynamics in the stenosis zone). Common and internal carotid arteries and vertebral and subclavian arteries were visualized from both sides during the ECA screening; common and deep femoral arteries and popliteal, anterior, and posterior tibial arteries were visualized from both sides during the LEA screening. The intima-media thickness (IMT) of the common carotid artery was measured in automatic mode (the value up to $1 \mathrm{~mm}$ was considered normal). Creatinine clearance was calculated using the Cockcroft-Gault equation: $\mathrm{CCr}=((140-$ Age $) \times$ mass (in kilograms $) \times[0.85$ if female $]) /(72 \times$ Serum Creatinine (in mg/dL)). Haemoglobin, total cholesterol, glucose, and fibrinogen levels were also estimated immediately after hospital admission and before discharge. The preferable methods of myocardial reperfusion were defined in the shortest terms and included percutaneous coronary intervention (PCI) and systemic thrombolytic therapy (TLT). Myocardial revascularization was not conducted when technical problems occurred or in patients with complex coronary anatomy or in those with contraindications to TLT or PCI. The clinical characteristics of the patients are presented in Table 1. All patients received the standard therapy of unfractionated heparin, aspirin, clopidogrel, angiotensin-converting enzyme inhibitors, beta-blockers, and statins. Reperfusion was not carried out in 157 (37.12\%) cases; additionally, 53 (12.53\%) patients received thrombolytic therapy, and 213 (50.35\%) underwent PCI. Statins, clopidogrel, aspirin, angiotensinconverting enzyme inhibitors, and beta-blockers were also
TABLE 1: Baseline characteristics.

\begin{tabular}{lc}
\hline & $\begin{array}{c}\text { ITT } \\
(n=423)\end{array}$ \\
\hline Mean age, mean years (95\% CI) & 61.77 \\
Male, \% & $60.68-62.87)$ \\
Prior angina pectoris, \% & $68.08 \%$ \\
Prior myocardial infarction, \% & $51.77 \%$ \\
Prior stroke, \% & $24.35 \%$ \\
Prior diabetes mellitus, \% & $8.51 \%$ \\
Prior arterial hypertension, \% & $18.20 \%$ \\
Smoking status, \% & $88.65 \%$ \\
Killip classes II-IV at admission, \% & $42.79 \%$ \\
\hline
\end{tabular}

prescribed after discharge. Additionally, nitrates, calcium channel blockers, and diuretics were prescribed to patients. Seven patients (1.65\%) died during hospitalisation. Coronary artery bypass surgery was not carried out during the hospitalization. The disease outcome in a 12-month follow-up period was defined in 397 patients (95.43\% of the initially discharged patients), and 39 fatal outcomes were registered (35 out of them were due to the cardiovascular causes; in the rest of the cases, the cause was not specified).

2.3. Statistical Analysis. A statistical analysis was conducted using IBM SPSS Statistic 19.0.0 (IBM Company, USA). We calculated the percentage ratio, mean, and the $95 \%$ confidence interval for the mean. The analysis of differences between the independent groups was carried out using Pearson's chi-square test. The Cox proportional hazards survival regression was applied to identify the independent predictors of the fatal outcomes. The value of the chi-square test and the area under the ROC-curve (AUC) were taken into consideration (C statistic). Missing data were not available during the procedure of multivariate analysis. Differences in the compared groups were considered to be statistically significant when the $P$ value was less than 0.05 .

\section{Results}

3.1. PVD Clinical Significance and Incidence. The prevalence of PVD, as defined by the increased intima-media thickness (IMT) or by the various degrees of stenosis, was quite high $(94.80 \%)$. In addition, $29.55 \%$ of the patients were characterised by stenosis with an occlusion of more than $50 \%$ of the vessel lumen, $17.73 \%$ of the patients were characterised by stenosis with an occlusion of $30 \%-50 \%$ of vessel lumen, and $44.52 \%$ of the patients were characterised by a stenosis with an occlusion of less than $30 \%$ of the vessel lumen or an increased IMT. Stratification of patients into groups revealed that the majority of patients (52.38\%) had lesions in all 3 arterial territories (coronary arteries, ECA, and LEA), 46.37\% of patients had CHD together with the lesions in the ECA, and only $1.25 \%$ of patients had manifestations of coronary artery atherosclerosis together with the lesions in the LEA. 
TABLE 2: Prevalence of risk factors among patients with STEMI according to the degree of stenosis in the noncoronary arteries (ECA or LEA).

\begin{tabular}{|c|c|c|c|c|c|}
\hline \multirow[b]{2}{*}{ Risk factors } & \multicolumn{4}{|c|}{ Patient groups } & \multirow[b]{2}{*}{$P$ value } \\
\hline & $\begin{array}{c}\mathrm{IMT}<1 \mathrm{~mm} \\
\text { No stenosis } \\
\quad(n=22)\end{array}$ & $\begin{array}{c}\mathrm{IMT} \geq 1 \mathrm{~mm} \\
\text { Stenosis }<30 \% \\
(n=201)\end{array}$ & $\begin{array}{l}\text { Stenosis } 30-50 \% \\
\quad(n=75)\end{array}$ & $\begin{array}{l}\text { Stenosis over } 50 \% \\
\quad(n=125)\end{array}$ & \\
\hline Age $>60$ years, $\%$ & 9.09 & 44.78 & 53.33 & 72.80 & $\begin{array}{l}\chi^{2}=42.12 \\
<0.0001\end{array}$ \\
\hline Prior angina pectoris, $\%$ & 22.73 & 48.26 & 45.33 & 66.40 & $\begin{array}{c}\chi^{2}=20.38 \\
0.0001\end{array}$ \\
\hline Prior MI, \% & 13.64 & 17.91 & 24.00 & 36.80 & $\begin{array}{c}\chi^{2}=16.42 \\
0.0009\end{array}$ \\
\hline Prior congestive HF, \% & 4.55 & 3.48 & 9.333 & 14.40 & $\begin{array}{l}\chi^{2}=13.35 \\
0.003\end{array}$ \\
\hline Prior stroke, $\%$ & 0 & 2.99 & 12.0 & 16.80 & $\begin{array}{l}\chi^{2}=22.13 \\
<0.0001\end{array}$ \\
\hline Killip II-IV, \% & 4.55 & 17.41 & 14.67 & 32.80 & $\begin{array}{l}\chi^{2}=17.56 \\
0.0005\end{array}$ \\
\hline $\begin{array}{l}\text { Glomerular filtration rate (MDRD formula) } \\
<60 \mathrm{~mL} / \mathrm{min} / 1.73 \mathrm{~m}^{2}, \%\end{array}$ & 3.4 & 42.5 & 24.8 & 29.3 & $\begin{array}{c}\chi^{2}=32.41 \\
0.0001\end{array}$ \\
\hline Concentration of triglycerides $>1.7 \mathrm{mmol} / \mathrm{L}$ & 73.68 & 54.04 & 45.16 & 35.35 & $\begin{array}{l}\chi^{2}=13.41 \\
0.003\end{array}$ \\
\hline $\mathrm{LV} \mathrm{EF} \leq 40 \%, \%$ & 9.09 & 12.44 & 18.67 & 25.60 & $\begin{array}{l}\chi^{2}=10.49 \\
0.014\end{array}$ \\
\hline CA lesions $>2, \%$ & 30.00 & 65.84 & 69.84 & 74.67 & $\begin{array}{c}\chi^{2}=14.54 \\
0.002\end{array}$ \\
\hline
\end{tabular}

IMT: intima-media thickness; MI: myocardial infarction; HF: heart failure; LV EF: left ventricular ejection fraction; CA: coronary artery.

Severe stenosis in the large arteries was more frequent among older patients and was associated with an increased incidence of angina pectoris, congestive heart failure, and prior myocardial infarction and stroke in the medical history. Acute heart failure (Killip II-IV), renal dysfunction, left ventricular systolic dysfunction, multivessel $\mathrm{CHD}$, and rare cases of hyperchylomicronaemia were registered in enrolled patients with PVD (Table 2). Thus, the presence of PVD among patients with STEMI was found to be associated with a more severe clinical status. This fact also increases the value of determining the pathological mechanisms, which may help to assess prognosis.

3.2. Prognostic Value of PVD. The GRACE score was calculated for all patients before discharge. On the basis of these scores, 167 subjects $(39.48 \%)$ were included in a low risk group (score 27-99), 140 individuals (33.10\%) were categorised in the average risk group (score 100-127), and 116 individuals $(27.42 \%)$ were regarded as high risk group (score $128-263$ ). The case fatality rates were $2.58 \%, 7.52 \%$, and $22.94 \%$, respectively, according to the distribution of GRACE score risk groups $\left(\chi^{2}=31.13, P<0.00001\right)$.

Stenosis of more than $30 \%$ of the ECA and LEA lumina was significantly associated with a high incidence of fatal outcomes within the one-year follow-up period $\left(\chi^{2}=13.68\right.$, $P=0.003)$. In addition, patients without $\operatorname{PVD}(n=18)$ had no fatal outcomes, in contrast to the patients with the ECA and LEA stenosis with stenosis of less than $30 \%$ of the vessel lumen $(n=193$, case fatality rate $5.70 \%)$. The individuals with stenosis with an occlusion of $30 \%-50 \%$ of the vessel lumen $(n=73)$ had $10.96 \%$ fatal outcomes in contrast to the patients with the stenosis of more than $50 \%$ of the vessel lumen ( $n=193$, case fatality rate $17.70 \%)$. There was no statistically significant difference in the incidence of fatal outcomes within the one-year follow-up period between patients who had lesions in all three vascular areas (coronary arteries, ECA, and LEA) and patients who had lesions only in two vascular areas (coronary arteries, ECA).

The Kaplan-Meier curve, showing the 12-month survival depending on the severity of peripheral artery disease, is shown in Figure 1. According to these data, the risk of death in patients with noncoronary artery stenosis with an occlusion of more than $30 \%$ of the vessel lumen was higher than that of other groups and increased steadily until the 12th month of followup.

3.3. Multiple Analysis Results and Prognostic Model Construction. We attempted to modify GRACE score model for the implementation of our results into the clinical practice. The Cox proportional hazards survival regression analysis with stepwise selection of independent risk factors for death was carried out for the achievement of this aim. The traditional risk factors (congestive heart failure, prior stroke, smoking status, and GRACE score) and the new risk factor, PVD with a varying degree of severity, were selected among various parameters (Table 3). Our obtained model of risk stratification included all the factors indicated in Table 3 and was named KemScore. The value of the $\chi^{2}$ model was 76.93 , 
TABLE 3: Variables in the regression equation (KemScore model).

\begin{tabular}{lcccc}
\hline Risk factors & $B$ & Wald & $P$ value & Exp $(B)(95 \%$ CI $)$ \\
\hline GRACE score & 0.65 & 5.26 & 0.022 & $1.91(1.13-3.35)$ \\
Congestive heart failure ((1) no, (2) yes) & 1.02 & 5.24 & 0.022 & $2.83(1.12-6.89)$ \\
Prior stroke ((1) no, (2) yes) & 1.40 & 13.79 & $<0.001$ & $4.14(1.88-8.52)$ \\
Smoking ((1) no, (2) yes) & 0.85 & 4.68 & 0.031 & $2.26(1.12-5.00)$ \\
PVD ((1) no, (2) stenosis $<30 \%$, and (3) stenosis $\geq 30 \%)$ & 1.30 & 17.28 & $<0.001$ & $3.73(2.00-6.81)$ \\
\hline
\end{tabular}

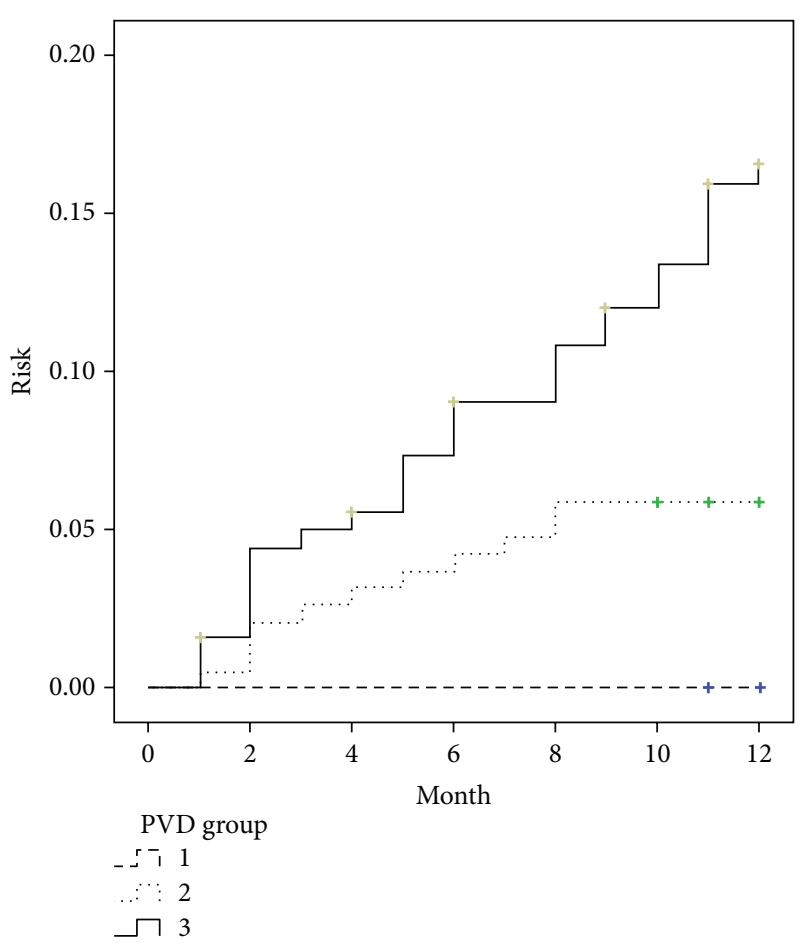

FIGURE 1: The risk of fatal outcome after myocardial infarction during a year of followup based on the Kaplan-Meier curve, according to the severity of noncoronary atherosclerosis: 1-no lesions of peripheral arteries, 2-ECA and LEA stenosis with an occlusion of less than $30 \%$ of the vessel lumen, and 3-ECA or LEA stenosis with an occlusion of more than $30 \%$ of the vessel lumen.

with $P<0.00001$. A comparative analysis of prognostic values of the new model (KemScore) and the GRACE score model was conducted by comparing the AUC (Figure 2).

The value of the AUC for the GRACE score model was 0.71 (95\% CI $=0.63-0.79)$, whereas this value for the KemScore model was 0.83 (95\% CI $=0.76-0.90)$. Thus, the addition of a new factor to the GRACE score model allows a more accurate risk stratification within one year after myocardial infarction. The previously described score system of risk factors was designed with the aim of convenient application in clinical practice (Table 4). A point value was assigned to each of the factors based on their influence on the risk of death. The case fatality rate after myocardial infarction, according to the scores within one year, is presented in Figure 3.

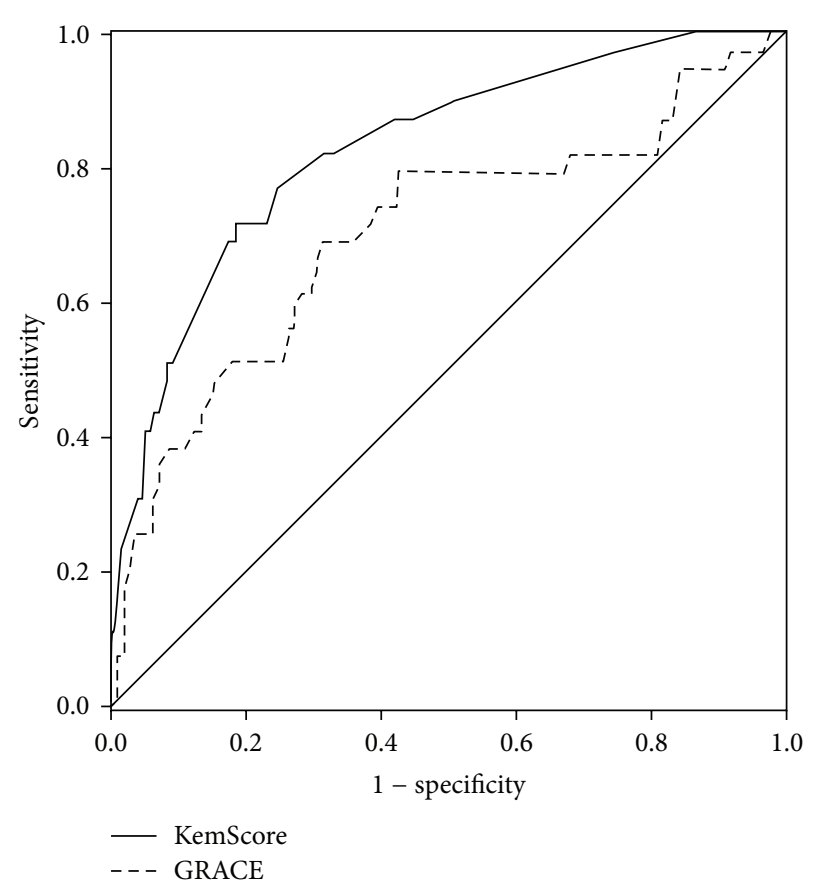

FIgURE 2: ROC curves of the GRACE score and KemScore.

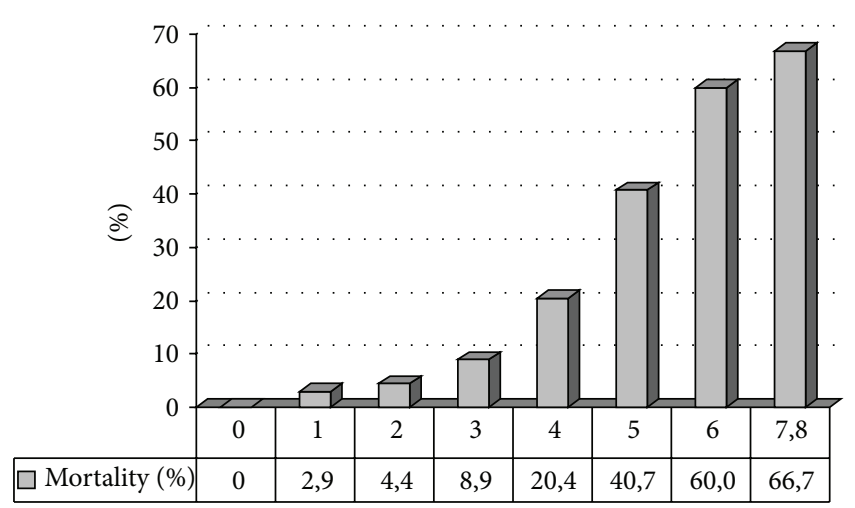

FIGURE 3: The case fatality rate according to the total score number in the KemScore model.

\section{Discussion}

Currently, there is no consensus on the incidence of multivessel atherosclerosis because of the different diagnostic approaches and study inclusion criteria. According to the literature, PVD incidence varies widely from $13.5 \%$ [6] to 
TABLE 4: Assessment of risk factors in cases with fatal outcomes within one year after myocardial infarction (KemScore model).

\begin{tabular}{llc}
\hline & Risk factors & Point \\
\hline $\begin{array}{l}\text { Risk of death after } \\
\text { discharge }\end{array}$ & $\begin{array}{l}\text { Low (27-99 scores) } \\
\text { Average (100-127 scores) }\end{array}$ & 0 \\
& High (128-263 scores) & 2 \\
\hline $\begin{array}{l}\text { Congestive heart } \\
\text { failure }\end{array}$ & No & 0 \\
\hline Prior stroke & Yes & 1 \\
\hline \multirow{2}{*}{ Smoking } & Yes & 0 \\
\hline \multirow{4}{*}{ Polyvascular diseases } & $\begin{array}{l}\text { No } \\
\text { Yes occlusion of less than } \\
\text { 30\% of the vessel lumen } \\
\text { ECA and LEA stenosis with } \\
\text { an occlusion of more than } \\
\text { 30\% of the vessel lumen }\end{array}$ & 2 \\
\hline
\end{tabular}

94\% [7]. Several authors assessed only clinical manifestations of ischemic vascular events $[8,9]$, while other authors used angiographic methods of diagnostics $[6,10]$, and still others focused on the ankle-brachial index [11] or ultrasound [7, 12] assessment methods. In addition, a wide range of PVD incidences in patients with varying degrees of cardiovascular risk can be explained by analysing subjects regardless of their age, gender, and ischemic heart disease forms (from stable angina to acute myocardial infarction with ST-segment elevation). However, it is obvious that patients examined in outpatient settings will be characterised as having relatively low cardiovascular risk, and a determination of PVD incidence is substantially complicated compared to patients admitted to the hospital as a result of various cardiovascular events who, therefore, had higher cardiovascular risk. The detection of stenosis in the ECA or LEA and increased IMT were the underlying criteria for the diagnosis of PVD in this study, and its prevalence was $95 \%$.

Undoubtedly, a case that presents with PVD determines the high risk status of a patient. However, it is impossible to determine the exact contribution of this particular condition to the risk stratification schemes at the present time, due to the different approaches in the diagnosis of peripheral artery lesions. A number of published studies also suggested that PVD patients constitute a high risk group. FerreiraGonzález et al. [4] have shown that patients with acute coronary syndrome associated with symptomatic peripheral arterial lesions had prior myocardial infarction, congestive heart failure, hypertension, hypercholesterolemia, diabetes mellitus, positive smoking status, and higher Killip class, and multivessel atherosclerosis of the coronary arteries is more often compared to individuals without such lesions, as also confirmed by Spencer et al. [6] and Van Kuijk et al. [13]. PVD was also associated with a higher frequency of adverse risk factors of presented coronary heart disease in this study.
According to Spencer et al. [6], a high case fatality rate was noted among patients with acute myocardial infarction and peripheral arterial disease (PAD) within 12 months of followup. Patients with PAD were at a significantly increased risk of death at 1 year after hospital discharge (adjusted OR $2.00,95 \% \mathrm{CI}=1.58-2.52)$ in comparison with patients without prior PAD [6]. In the CAFES-CAVE prospective study [14], 13,221 patients without clinical manifestations of cardiovascular diseases and with low risk of their development were involved. All patients were divided into four groups according to the results of ultrasonography of the extracranial arteries and lower extremity arteries. Group 1 had no pathological changes in the arteries, Group 2 had high IMT, Group 3 had plaques without luminal stenosis, and Group 4 had plaques with luminal stenosis. The prevalence of adverse events over several years of followup was $0.1 \%$, $8.6 \%, 39.3 \%$, and $81.1 \%$ in these four groups, respectively. The authors supposed high IMT to be associated with a low risk of cardiovascular events, the plaques without luminal stenosis to be associated with a moderate risk, and the plaques with luminal stenosis to be correlated with a high risk. In our study, patients with STEMI, even with haemodynamically insignificant stenosis of noncoronary vascular origin, were shown to have significantly worse outcomes after 12 months of followup compared to patients with STEMI without PVD.

The past literature has shown evidence to support the idea that the GRACE score is equally effective in assessing prognosis at 6 and 12 months after acute coronary syndrome [15]. De Araújo Gonçalves et al. [15] studied 460 consecutive patients admitted to the coronary care unit with an ACS. The best predictive accuracy for death or MI at 1 year was obtained by the GRACE (AUC: $0.72(95 \% \mathrm{CI}=0.67-0.76))$ [15]. This has also been confirmed by the present study. The values of the AUC for the prognosis assessment ability of the GRACE score at the 6- and 12-month follow-up period were 0.73 (95\% $\mathrm{CI}=0.64-0.82)$ and $0.71(95 \% \mathrm{CI}=0.63-0.79)$, respectively. In the study by Abu-Assi et al. [16] on 1183 Spanish patients, the predictive value of the GRACE score was significantly higher, as the area under the curve (AUC) was 0.90 (95\% CI = $0.83-0.98$ ). However, in the study by Lev et al. [3] on 974 Israeli patients, this value was much lower (AUC was 0.50 $(95 \% \mathrm{CI}=0.47-0.54))$. These examples show restrictions in the applicability of the current GRACE score model and the necessity to improve this model by adding new parameters. This study, therefore, proposed an improvement in the ability of the current model to predict prognosis by adding the severity of polyvascular diseases as an additional risk factor to the GRACE risk score. This factor is suggested to reflect not only a variety of other cardiovascular risk factors but also the severity of subclinical inflammation. It is important because such subclinical inflammation may lead to progression of atherosclerosis and the destabilisation of haemodynamically insignificant atherosclerotic plaques, causing development of new vascular events.

Unfortunately, our study has several limitations. Firstly, the sample size was not large; secondly, we have not assessed the reclassification and other endpoints such as nonfatal myocardial infarction, stroke, and so forth. 


\section{Summary}

Polyvascular diseases with even insignificant stenosis of the peripheral arteries not only determine the presence of multiple risk factors for cardiovascular diseases in patients with myocardial infarction but also appear to be an independent predictor of adverse (fatal) cardiovascular outcome. Therefore, this factor should be considered in the risk stratification.

\section{Conflict of Interests}

The authors declare that they have no conflict of interests.

\section{References}

[1] D. A. Morrow, E. M. Antman, A. Charlesworth et al., "TIMI risk score for ST-elevation myocardial infarction: a convenient, bedside, clinical score for risk assessment at presentation: an Intravenous nPA for Treatment of Infarcting Myocardium Early II trial substudy," Circulation, vol. 102, no. 17, pp. 2031-2037, 2000.

[2] K. A. A. Fox, O. H. Dabbous, R. J. Goldberg et al., "Prediction of risk of death and myocardial infarction in the six months after presentation with acute coronary syndrome: prospective multinational observational study (GRACE)," British Medical Journal, vol. 333, no. 7578, pp. 1091-1094, 2006.

[3] E. I. Lev, R. Kornowski, H. Vaknin-Assa et al., "Comparison of the predictive value of four different risk scores for outcomes of patients with ST-elevation acute myocardial infarction undergoing primary percutaneous coronary intervention," American Journal of Cardiology, vol. 102, no. 1, pp. 6-11, 2008.

[4] I. Ferreira-González, G. Permanyer Miralda, M. Heras et al., "Prognosis and management of patients with acute coronary syndrome and polyvascular disease," Revista Española de Cardiología, vol. 62, pp. 1012-1021, 2009.

[5] F. Van de Werf, J. Bax, A. Betriu et al., "Management of acute myocardial infarction in patients presenting with persistent STsegment elevation," European Heart Journal, vol. 29, pp. 29092945, 2008.

[6] F. A. Spencer, D. Lessard, C. Doubeni, J. Yarzebski, J. M. Gore, and R. J. Goldberg, "Treatment practices and outcomes of patients with established peripheral arterial disease hospitalized with acute myocardial infarction in a community setting," American Heart Journal, vol. 153, no. 1, pp. 140-146, 2007.

[7] A. Kablak-Ziembicka, W. Tracz, T. Przewlocki, P. Pieniazek, A. Sokolowski, and M. Konieczynska, "Association of increased carotid intima-media thickness with the extent of coronary artery disease," Heart, vol. 90, no. 11, pp. 1286-1290, 2004.

[8] S. Coccheri, "Distribution of symptomatic atherothrombosis and influence of atherosclerotic disease burden on risk of secondary ischaemic events: results from CAPRIE," European Heart Journal, vol. 19, article 1268, 1998.

[9] D. L. Bhatt, P. Gabriel Steg, E. Magnus Ohman et al., "International prevalence, recognition, and treatment of cardiovascular risk factors in outpatients with atherothrombosis," Journal of the American Medical Association, vol. 295, no. 2, pp. 180-189, 2006.

[10] R. A. Aqel, G. J. Zoghbi, S. A. Baldwin et al., "Prevalence of renal artery stenosis in high-risk veterans referred to cardiac catheterization," Journal of Hypertension, vol. 21, no. 6, pp. 11571162, 2003.
[11] P. Poredoš and B. Jug, “The prevalence of peripheral arterial disease in high risk subjects and coronary or cerebrovascular patients," Angiology, vol. 58, no. 3, pp. 309-315, 2007.

[12] D. Calvet, E. Touzé, O. Varenne, J.-L. Sablayrolles, S. Weber, and J.-L. Mas, "Prevalence of asymptomatic coronary artery disease in ischemic stroke patients: the precoris study," Circulation, vol. 121, no. 14, pp. 1623-1629, 2010.

[13] J.-P. Van Kuijk, W.-J. Flu, G. M. J. M. Welten et al., "Long-term prognosis of patients with peripheral arterial disease with or without polyvascular atherosclerotic disease," European Heart Journal, vol. 31, no. 8, pp. 992-999, 2010.

[14] G. Belcaro, A. N. Nicolaides, G. Ramaswami et al., "Carotid and femoral ultrasound morphology screening and cardiovascular events in low risk subjects: a 10-year follow-up study (the CAFES-CAVE study)," Atherosclerosis, vol. 156, no. 2, pp. 379$387,2001$.

[15] P. De Araújo Gonçalves, J. Ferreira, C. Aguiar, and R. SeabraGomes, "TIMI, PURSUIT, and GRACE risk scores: sustained prognostic value and interaction with revascularization in NSTE-ACS," European Heart Journal, vol. 26, no. 9, pp. 865-872, 2005.

[16] E. Abu-Assi, J. M. García-Acuña, C. Peña-Gil, and J. R. González-Juanatey, "Validation of the GRACE risk score for predicting death within 6 months of follow-up in a contemporary cohort of patients with acute coronary syndrome," Revista Española de Cardiología, vol. 63, pp. 640-648, 2010. 


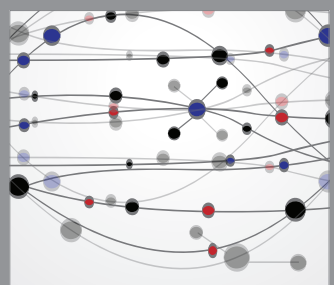

The Scientific World Journal
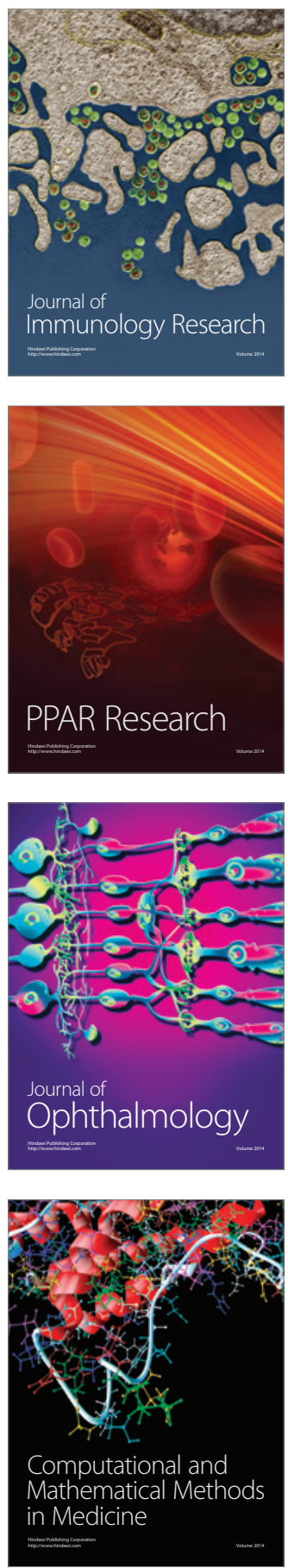

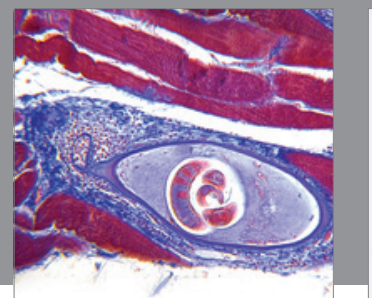

Gastroenterology

Research and Practice
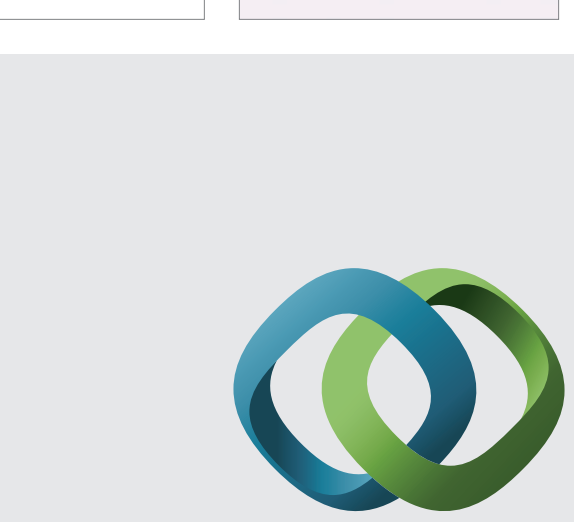

\section{Hindawi}

Submit your manuscripts at

http://www.hindawi.com
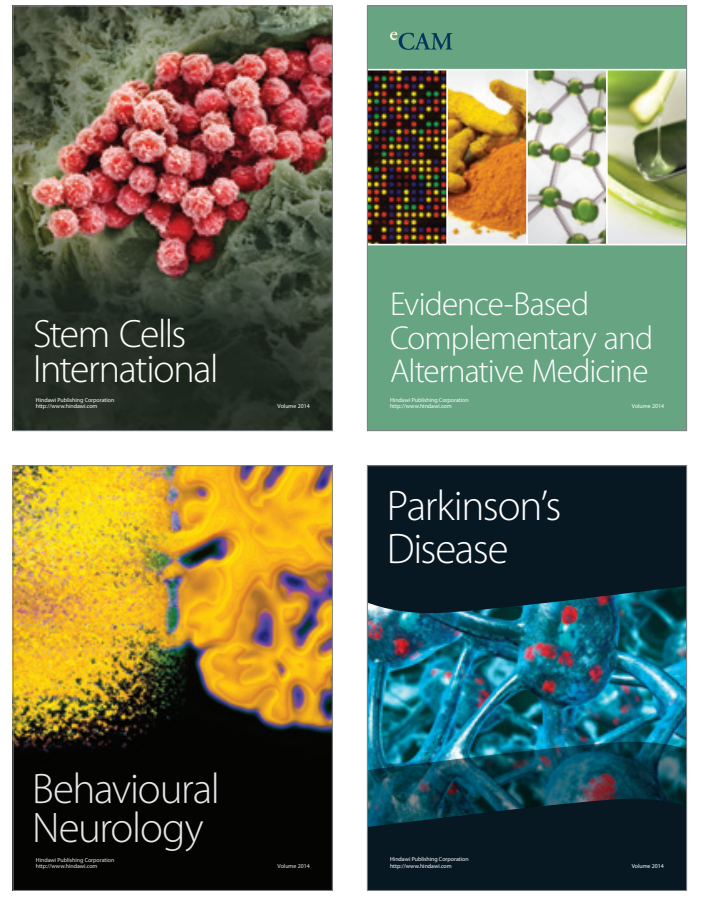
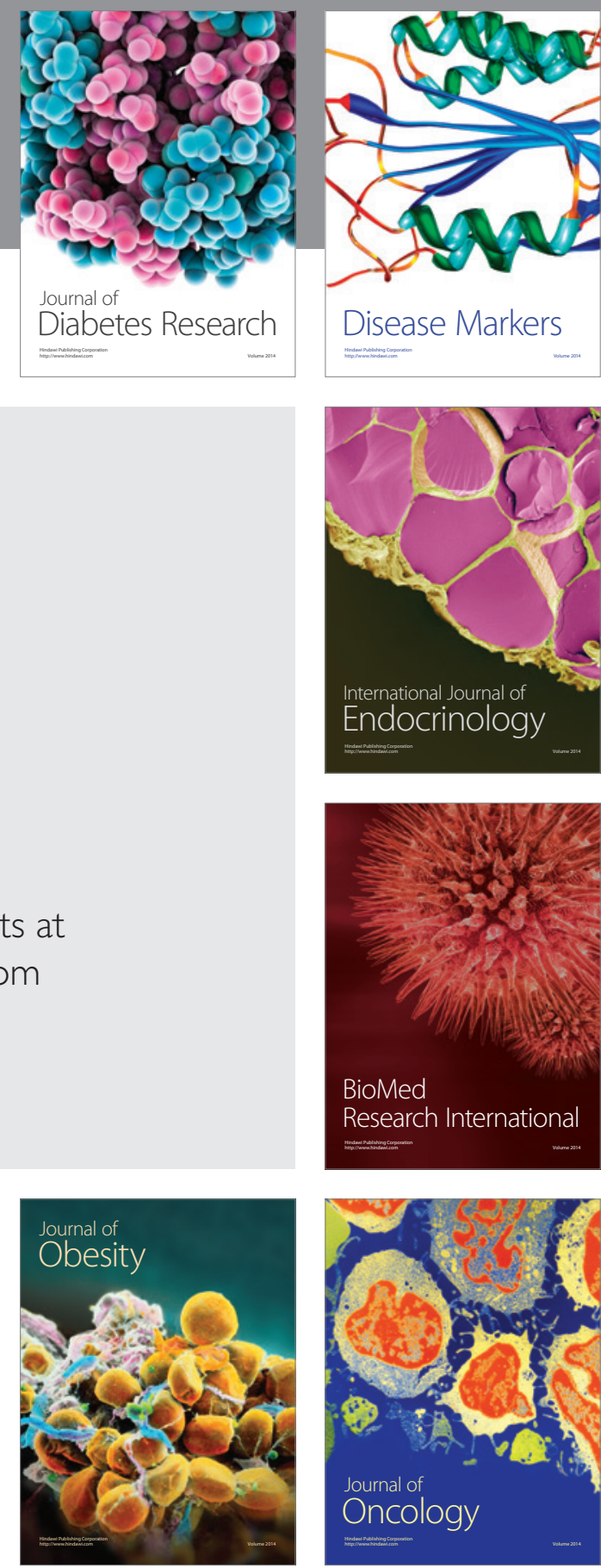

Disease Markers
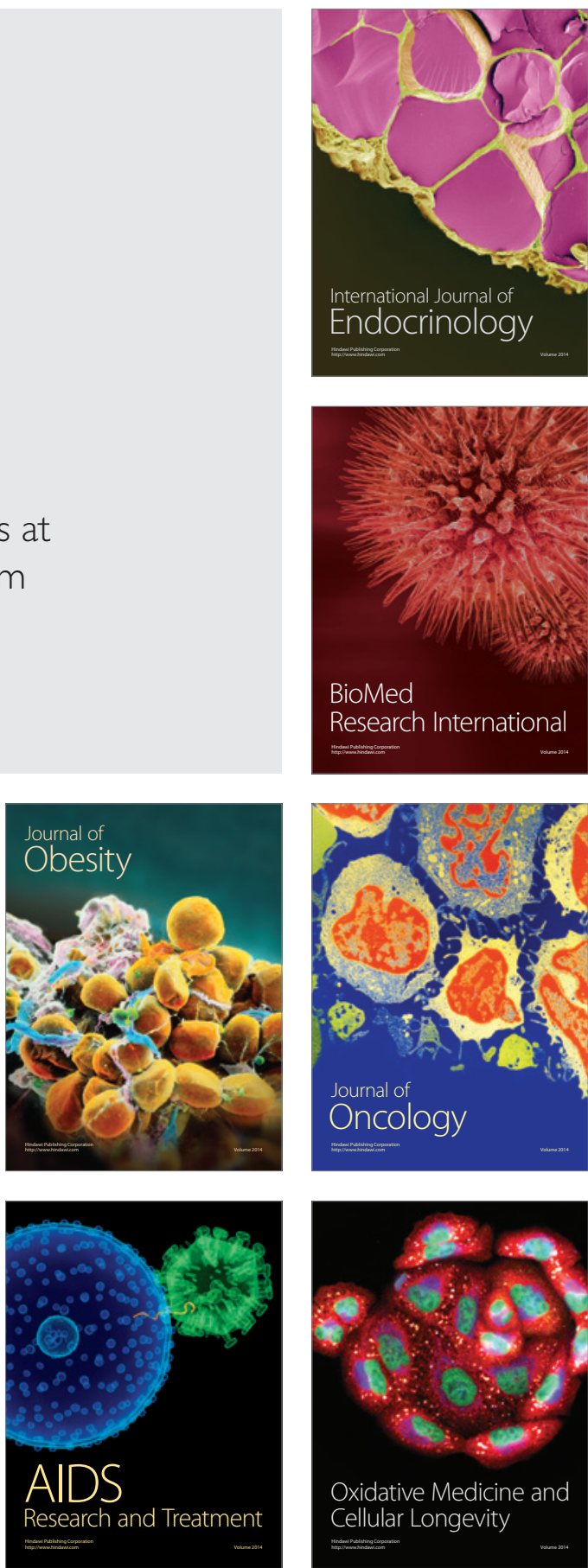